\title{
Dual energy 4D-CT of parathyroid adenomas not clearly localized by sestamibi scintigraphy and ultrasonography - a retrospective study
}

Mischa Woisetschläger, Oliver Gimm, K. Johansson, G. Wallin, I Albert-Garcia and Anna Spångeus

The self-archived postprint version of this journal article is available at Linköping University Institutional Repository (DiVA):

http://urn.kb.se/resolve?urn=urn:nbn:se:liu:diva-164169

N.B.: When citing this work, cite the original publication.

Woisetschläger, M., Gimm, O., Johansson, K., Wallin, G., Albert-Garcia, I, Spångeus, A., (2020), Dual energy 4D-CT of parathyroid adenomas not clearly localized by sestamibi scintigraphy and ultrasonography - a retrospective study, European Journal of Radiology, 124, 108821.

https://doi.org/10.1016/j.ejrad.2020.108821

Original publication available at:

https://doi.org/10.1016/j.ejrad.2020.108821

Copyright: Elsevier (12 months)

http://www.elsevier.com/

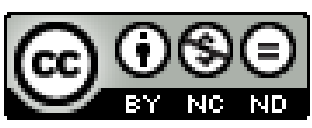




\section{Journal Pre-proof}

Dual energy 4D-CT of parathyroid adenomas not clearly localized by sestamibi scintigraphy and ultrasonography - a retrospective study

M. Woisetschläger, O. Gimm, K. Johansson, G. Wallin, I.

Albert-Garcia, A. Spångeus

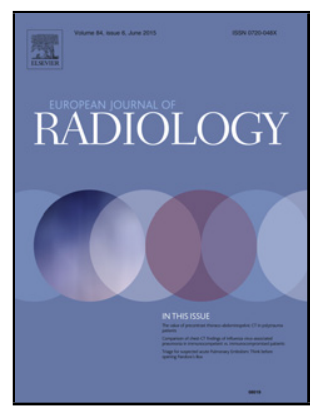

$\begin{array}{ll}\text { PII: } & \text { S0720-048X(20)30010-3 } \\ \text { DOI: } & \text { https://doi.org/10.1016/j.ejrad.2020 } \\ \text { Reference: } & \text { EURR 108821 } \\ \text { To appear in: } & \text { European Journal of Radiology } \\ \text { Received Date: } & 27 \text { March } 2019 \\ \text { Revised Date: } & 17 \text { December } 2019 \\ \text { Accepted Date: } & \text { 30 December } 2019\end{array}$

Please cite this article as: Woisetschläger M, Gimm O, Johansson K, Wallin G, Albert-Garcia I, Spångeus A, Dual energy 4D-CT of parathyroid adenomas not clearly localized by sestamibi scintigraphy and ultrasonography - a retrospective study, European Journal of Radiology (2020), doi: https://doi.org/10.1016/j.ejrad.2020.108821

This is a PDF file of an article that has undergone enhancements after acceptance, such as the addition of a cover page and metadata, and formatting for readability, but it is not yet the definitive version of record. This version will undergo additional copyediting, typesetting and review before it is published in its final form, but we are providing this version to give early visibility of the article. Please note that, during the production process, errors may be discovered which could affect the content, and all legal disclaimers that apply to the journal pertain.

(c) 2019 Published by Elsevier. 


\section{Dual energy 4D-CT of parathyroid adenomas not clearly localized by sestamibi scintigraphy and ultrasonography - a retrospective study}

Short title/running title: Dual energy 4D-CT - characterization of parathyroid adenomas

M. Woisetschläger ${ }^{a, b}$, O. Gimmc, K. Johansson ${ }^{d}$, G. Wallin ${ }^{e}$, I. Albert-Garciaa,b, A. Spångeus ${ }^{f}$

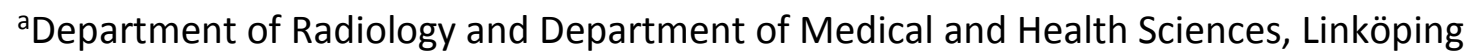

${ }^{b}$ Center for Medical Image Science and Visualization (CMIV), Linköping University, Linköping, Sweden

'Department of Surgery and Department of Clinical and Experimental Medicine, Linköping University, Linköping, Sweden

${ }^{\mathrm{d} D e p a r t m e n t}$ of Surgery Västervik and Faculty of Medicine and Health, Örebro University Hospital

eFaculty of Medicine and Health, Örebro University Hospital, Örebro

fDepartment of Endocrinology and Department of Medical and Health Sciences, Linköping University, Linköping, Sweden

Correspondence: Mischa Woisetschläger, MD, PhD, Department of Radiology, Linköping, Building 411, level 11, University hospital Linköping, 58185 Linköping, Sweden. Mischa.woisetschlager@regionostergotland.se, Phone: +46 101032728

Contact details:

Mischa Woisetschläger

Department of Radiology

Linköping, Building 411, level 11

University hospital Linköping, 58185 Linköping, Sweden

Mischa.woisetschlager@regionostergotland.se

Phone: +46 101032728

Oliver Gimm

Department of Surgery 
Linköping, Building 411, level 14

University hospital Linköping, 58185 Linköping, Sweden

oliver.gimm@regionostergotland.se

Phone: +46101038041

Kenth Johansson

Department of Surgery

Västervik hospital, Östra Kyrkogatan 48, Västervik

kenth.johansson@regionkalmar.se

phone: $+46490-86000$

Göran Wallin

Dept of Surgery

Örebro University Hospital, SE-701 85 Örebro

goran.wallin@regionorebrolan.se

+46 (0)19602 1370/1000

Isidro Albert-Garcia

Department of Radiology

Linköping, Building 411, level 11

University hospital Linköping, 58185 Linköping, Sweden

Isidro.Albert.Garcia@regionostergotland.se

Phone: +46101030000

Anna Spångeus

Department of Medical and Health Sciences

University Linköping, 58183 Linköping, Sweden

Anna.spangeus@liu.se 
Highlights

- Diagnostic accuracy of parathyroid adenoma could be enhanced using dual-energy CT

- Dual-energy CT from real non-contrast scans could help in equivocal cases

- Lower energies showed greater HU differences in spectral analysis

\section{Abstract}

Purpose: At present, the gold standard for diagnosing PAs includes ultrasonography of the neck and sestamibi scans of the parathyroid. The objective of this study was to evaluate scans performed in 4D-DECT (4D-dual-energy mode) at three different time points, in order to analyze spectral information from PAs, lymph nodes (LNs), and thyroid gland (Thy).

Method: Fifteen patients (mean age: $57 \pm 18.9$ years) with primary hyperparathyroidism, in which previous ultrasound and sestamibi scanning proved to be negative or equivocal, underwent 4D-DECT in three different phases. Hounsfield units (HU), dual-energy information (electron density [Rho], atomic number [Z], dual-energy index [DEI]), and spectral information (keV) were determined.

Results: For all energies, PAs exhibited significantly lower HU-values than the Thy in noncontrast images, and higher HU-values than LNs in the arterial phase $(p<0.05)$. All three tissues differed significantly in $\mathrm{HU}$ in the venous phase at $90 \mathrm{kV}, 150 \mathrm{kV}$, and mixed 0.8 images; the Thy showed significantly higher HU-values than PAs or LNs in non-contrast images at $90 \mathrm{kV}$, $150 \mathrm{kV}$, mixed 0.8 images, and [Rho] ( $\mathrm{p}<0.05)$. LNs exhibited significantly lower HU-values than 
PAs and Thy in the arterial phase at $90 \mathrm{kV}, 150 \mathrm{kV}$, mixed 0.8, Rho, Z, and DEI $(p<0.05)$. With regards to spectral information, lower energies showed greater HU differences between the three tissues. During the venous phase, there were significant differences between all three tissues up to $100 \mathrm{keV}(\mathrm{p}<0.05)$.

Conclusions: We identified significant differences in HU-values and spectral information between PAs, LNs, and Thy at different energies and contrast phases.

Keywords: parathyroid adenoma; computed tomography; dual-energy CT; 4D-CT; quantitative information

\section{Introduction}

The main function of the parathyroid glands is to regulate calcium balance in the body. This is important for many processes, including nerve and muscle function, and development of the skeleton. Usually, these four glands are located close to the thyroid gland, although in some cases, they can be located in ectopic regions, ranging from the base of the skull, to the height of the carina. Primary hyperparathyroidism (pHPT) is a common disease; a recent study showed that the prevalence of pHPT in a Swedish population of postmenopausal women was 3.4\% [1]. In pHPT, one or more parathyroid gland adenomas produce excessive levels of parathormone (PTH), thus leading to hypercalcemia.

The treatment of choice for pHPT is surgery; the complication rate is low and the risk of surgical mortality is almost non-existent [2]. Prior to surgery it is important to localize any enlarged, and presumably overactive, parathyroid glands. For this purpose, our institution routinely performs ultrasonography on the neck, as well as parathyroid sestamibi scintigraphy. 
For unclear cases, or for cases involving persistent/recurrent pHPT following surgery, physicians may also perform four dimensional computed-tomography (4D-DECT) using a standardized protocol with three different phases.

The principle underlying the use of 4D-CT to identify and localize parathyroid adenomas relies on differences in the perfusion characteristics of parathyroid adenomas in comparison with adjacent structures, such as the thyroid gland (Thy) and lymph nodes (LNs). Parathyroid adenomas (PAs) usually show similar attenuation values (Hounsfield units [HU]) in noncontrast examinations compared to LNs, lower HU values compared to the Thy, and a clear enhancement pattern in the arterial phase with washout in the venous phase $[3,4]$. Until now, only a small number of studies have assessed the dual-energy technique for its ability to evaluate PAs [5-11]. These previous studies concluded that quantitative information related to dual-energy scans might help in distinguishing between tissues in the arterial and venous phase.

To our knowledge, no previous study has described the quantitative analysis of dual-energy non-contrast scans, or investigated atomic numbers, electron density, dual-energy index (DEI), and spectral information, in non-contrast dual-energy CT scans from patients with persistent/recurrent PHPT. Consequently, we aimed to evaluate scans performed in 4D-DECT at three different time points and differing contrast enhancements, and to analyze spectral information from PAs, LNs, and the Thy.

\section{Materials and methods}

\section{Patients}

This retrospective study included all patients with hyperparathyroidism from our hospital, and nearby hospitals, who underwent pre-surgery 4D-DECT at our department between 2012 and 
2018. All cases subsequently underwent successful parathyroidectomy (the diagnosis of PA was confirmed surgically and pathologically). In these cases, standard pre-surgery imaging techniques (ultrasound and sestamibi scintigraphy) were unable to clearly identify PAs. Consequently, 4D-DECT was applied.

\section{CT technique}

Two patients were scanned with a Siemens Somatom Definition Flash and Stellar detector (Somatom Definition Flash; Siemens Healthcare, Erlangen, Germany), while 13 patients were scanned with a Siemens Somatom Force scanner (Somatom Force, Siemens Healthcare, Erlangen). Scans were obtained in dual-energy mode (100/Sn140 for the Flash; 90/Sn150 for the Force) in three phases; 1) without contrast ('w/o'), 2) arterial phase ('art'), and 3) the venous phase ('ven'). Bolus tracking was used for the contrast phases, with a 10 second delay before tracking commenced, in the descending aorta of the thorax with a threshold of $130 \mathrm{HU}$ (which took approximately 5 seconds), and a delay of 8 seconds before the arterial scan was carried out. The arterial scan was carried out 30 seconds after the contrast bolus was administered and took approximately 4 seconds to complete. Forty-five seconds after the arterial scan was completed, the venous scan was carried out (approximately 75 seconds after the contrast bolus was administered). The concentration of the contrast agent (Ultravist, Bayer) was $370 \mathrm{mgl} / \mathrm{ml}$; the standard dose was $450 \mathrm{mgl} / \mathrm{kg}$. The contrast agent was administered over 18 seconds.

Rotation time was 0.25 , with a pitch of 0.7 , and an approximate scan length of $35 \mathrm{~cm}$ (from the base of the skull to just under the carina. The field of view (FoV) was $500 \mathrm{~mm}$ and the images were reconstructed with two different slice thicknesses: 1) $0.75 \mathrm{~mm}$ and an increment of $0.7 \mathrm{~mm}$ for the quantitative analysis in syngo.Via (Siemens Healthcare, Erlangen, Version 
VB30A_HF02), and 2) with a slice thickness of $3 \mathrm{~mm}$ and a $3 \mathrm{~mm}$ increment (3/3) for visual assessment. The $3 / 3$ had a dual-energy composition (DE) of 0.8 for all three phases. These images were subsequently transferred to the Picture Archive and Communication System (PACS) and used for clinical evaluation.

\section{Image analysis}

For each patient, we documented the number of PAs, their location, and their size. Two experienced radiologists ( $M W$ \& IAG) determined whether the color-coded images in the Monoenergetic+ module led to a change in the results (1: no difference; 2 : increased somewhat; 3: increased a lot; 4: additional adenomas found). We also documented whether the adenomas were to the right or left of the patient's midline. The specific criteria used to diagnose PA included an intense enhancement on arterial phase images, with washout on the venous phase and low attenuation on non-contrast images. We also considered a number of secondary signs, including enlarged feeding arteries, or draining veins to the hypervascular structure, as well as signs of cystic changes.

Quantitative evaluation was performed in Syngo.Via (Siemens Healthcare, Erlangen, Version VB30A_HF02); the stacks were first loaded into the dual-energy module and then into the monoenergetic+ module. PAs, LNs, and the Thy, were each evaluated in all three phases; we tried to retain the same region of interest (ROI) size for all three structures in the same patient. For PAs, the ROI included the enhanced area of the structure but avoided heterogeneous and cystic structures. For LNs, we preferred to use nearby nodes from regions IV and VI. If we failed to detect LNs in region IV and V, then a level I or II LN was taken. Only non-pathological LNs were analyzed; we excluded LNs with a short axis diameter exceeding $10 \mathrm{~mm}$, or those with 
irregular borders and internal heterogeneity. For the Thy tissue, we only included tissue with a normal appearance in our evaluations. All ROls were placed by an experienced radiologist (MW).

All three structures (PAs, LNs, and the Thy) were measured in all three phases (w/o, art, ven) using $90 \mathrm{kV}, 150 \mathrm{kV}$, and mixed images (DE composition factor: 0.8). For each structure, we recorded the $\mathrm{HU}$ value. We also measured electron density (Rho), atomic number (Z), and dual-energy index (DEI). We obtained spectral HU attenuation curves for all three structures, and all three phases, in the monoenergetic+ module, ranging from $40 \mathrm{keV}$ to $190 \mathrm{keV}$.

Using these parameters, we calculated representative values for each tissue and phase. For Z values and $\mathrm{DEI}$, the measurement values were taken directly from the Syngo.Via application. For Rho, the correct value had to be calculated, as the Syngo.Via application only provides Rho $\mathrm{HU}\left(\mathrm{HU}_{\mathrm{x}}\right)$. In order to determine the true electron density, $\mathrm{HU}_{\mathrm{x}}$ had to be divided by 1000 and then 1 had to be added. This value was then multiplied by the density of water $\left(3.35 \times 10^{-23}\right)$ in order to normalize against the density of water. (Equation 1)

Equation 1:

$$
\sigma=\left(\left(\frac{\mathrm{HUx}}{1000}\right)+1\right) * 3.35 * 10^{-23}
$$

\section{Radiation dose}

The reference mAs for scans carried out at 100/Sn140 Kv and 90/Sn150 Kv were 104 mAs and $90 \mathrm{mAs}$, respectively. The effective dose was calculated by dividing the total DLP (dose length product) by 2 and multiplying one part by 0.0059 (neck), and one part by 0.014 (thorax). The 
sum of the two products represented the total sum of the effective dose for the examination, taking into account the different $\mathrm{k}$ factors for the organs.

\section{Statistical analysis}

One-way analysis of variance (ANOVA) was used, along with a Bonferroni post-hoc test, to analyze the differences between PAs, LNs, and the Thy. A p-value less than 0.05 was considered to be statistically significant. All statistical analysis was carried out using SPSS (IBM SPSS Statistics version 23.0, Chicago, USA).

\section{Ethics}

The study was approved by the regional ethics board. (Reference Number: 2017/241-31).

\section{Results}

\section{Patient characteristics and radiation dose}

In total, 15 consecutive patients ( 9 women and 6 men) were included in this study. The mean age was $57 \pm 18.9$ years (range: $22-82$ years). The median (range) of the ionized calcium concentration in the blood (mmol/l) was 1.45 (range 1.34-1.69) preoperatively, 1.23 (range 1.05-1.43) immediately post-operatively, and 1.27 (range 1.17-1.37) at 6 months after operation. The corresponding values for PTH (pmol/l) were 14.0 (5.3-41), 1.7 (0.6-5.6), and $3.3(0.6-8.8)$. Normal reference values are $1.15-1.33 \mathrm{mmol} / \mathrm{l}$ (ionized calcium) and 1.6-6.9 pmol/I (PTH). One patient was analyzed pre-operatively using another method with a higher reference interval; this patient's PTH value was 116 (reference range: $15-65 \mathrm{ng} / \mathrm{l}$ ). 
The mean radiation dose was $803 \mathrm{mGycm}$ (DLP), and the effective dose was $7.99 \mathrm{mSv}$ (range: 6.4-10.7 mSv). The mean volume of contrast media applied was $95 \mathrm{ml}$ (range: $70-113 \mathrm{ml}$ ), with a mean flow rate of $4.8 \mathrm{ml} / \mathrm{s}$ (range: $3.9-6.2 \mathrm{ml} / \mathrm{s}$ ).

\section{Radiological location}

Three PAs (13\%) were located dorsally to the upper quadrants of the thyroid; two on the right side and one on the left side. Seven PAs (47\%) were located dorsally to the lower quadrants of the thyroid; five on the right side and two on the left side. One (6.7\%) PA was located in the superior thymus on the left side. One PA (6.7\%) was located in an intrathyroidal position on the right side. Four PAs (26.7\%) were located ectopically; two in the mediastinum on the left side and under the aortic arch in the midline, one associated with an intrathyroidal cyst on the right side, and one located pre-tracheally in the sternal notch in the midline. In total, nine PAs were located on the right side, five on the left side, and two in the midline. One patient had two PAs (one in the left caudal quadrant, and one pre-tracheally in the sternal notch), while the remaining patients only had one PA on either the left or the right side.

The mean size of the PAs identified was $9.6 \mathrm{~mm}$ (range: 4-19 mm), with 10 PAs measuring 10 $\mathrm{mm}$ or less. Figure 1 shows an example of one of the patients illustrating the key differences between contrast phases and the evaluation methods.

\section{Color mapping of Rho/Z}

Color mapping of the Rho/Z module increased the levels of diagnostic confidence in eight of the 15 patients. In these eight patients, the image reviewers stated that color mapping increased the diagnostic confidence 'a lot' in two cases and 'somewhat' in six cases. The reviewers stated that the main benefit of color mapping was that smaller PAs were easier to 
detect. No major value was added in terms of distinguishing between different tissues. The reviewers did not identify any additional adenomas.

HU data at 90 and $150 \mathrm{Kv}$, and mixed images in all three phases

As shown in Figure $2 \mathrm{~A}-\mathrm{C}$, all of the energies examined $(90 \mathrm{kV}, 150 \mathrm{kV}$, and mixed images 0.8) showed similar patterns of discrimination between the three tissues. The pattern of contrast uptake showed low HU values for PAs and LNs without contrast, and higher HU values for the Thy. In the arterial phase, the Thy and PAs showed a clear contrast uptake; the Thy showed higher uptake rates than PAs. LNs showed only a slight to moderate uptake in the arterial phase. In the venous phase, the Thy and PAs showed lower HU values (washout) when compared with the arterial phase, although LNs showed a slightly higher uptake compared to the arterial phase.

In general, the absolute $\mathrm{HU}$ values showed larger differences at $90 \mathrm{kV}$ than at mixed 0.8 and $150 \mathrm{kV}$. Thus, the mean differences in $\mathrm{HU}$ values between the Thy and PAs at $90 \mathrm{kV}$ were 51 $\mathrm{HU}$ (non-contrast), $50 \mathrm{HU}$ (arterial), and $43 \mathrm{HU}$ (venous); at mixed 0.8, these values were 46 $\mathrm{HU}$ (non-contrast), $39 \mathrm{HU}$ (arterial), and $39 \mathrm{HU}$ (venous). At $150 \mathrm{kV}$; these values were $37 \mathrm{HU}$ (non-contrast), $24 \mathrm{HU}$ (arterial), and $32 \mathrm{HU}$ (venous). Similarly, the mean differences in HU between PAs and LNs at $90 \mathrm{kV}$ were: $-7 \mathrm{HU}$ (non-contrast), $149 \mathrm{HU}$ (arterial), and $43 \mathrm{HU}$ (venous). At mixed 0.8, these values were $-5 \mathrm{HU}$ (non-contrast), $126 \mathrm{HU}$ (arterial), and $36 \mathrm{HU}$ (venous). At $150 \mathrm{kV}$, these values were $-2 \mathrm{HU}$ (non-contrast), $62 \mathrm{HU}$ (arterial), and $16 \mathrm{HU}$ (venous). 


\section{Electron density Rho (O') in all three phases}

As shown in Figure 3A, the Rho of the Thy was significantly higher than PAs and LNs in all three contrast phases $(p<0.05)$. Parathyroid adenomas had a significantly higher Rho value than LNs in the arterial phase $(p<0.05)$, although there was no significant difference in the venous phase or without contrast. Rho values for PA were $3.41 \pm 0.07 \times 10^{-23}$ (non-contrast), $3.49 \pm 0.06 \times 10^{-}$ ${ }^{23}$ (arterial), and $3.46 \pm 0.07 \times 10^{-23}$ (venous). Rho was significantly higher in the arterial phase than in non-contrast $(p=0.016)(p<0.05)$, although there were no other significant differences between the other phases. Corresponding values for the Thy were $3.52 \pm 0.04 \times 10^{-23}$ (noncontrast), $3.54 \pm 0.04 \times 10^{-23}$ (arterial), and $3.54 \pm 0.03 \times 10^{-23}$ (venous). For LNs, these values were $3.44 \pm 0.03 \times 10^{-23}$ (non-contrast), $3.44 \pm 0.04 \times 10^{-23}$ (arterial), and $3.45 \pm 0.04 \times 10^{-23}$ (venous). For the Thy and LNs, Rho did not differ significantly between contrast phases.

\section{$Z$ in all three phases}

In arterial and venous phases, LNs showed significantly lower values for $Z$ than both PAs and the Thy (Figure 3B) $(p<0.05)$. No significant differences were detected between PAs and the Thy in any contrast phase.

\section{$D E I$ in all three phases}

Similar to the $Z$ value, DEI was significantly lower in LNs than in PAs and the Thy in arterial and venous phases $(p<0.05)$, but there was no statistical difference between PAs and the Thy. Without contrast, there was no statistical difference between the three tissues (Figure 3C). 
In the non-contrast phase, the Thy showed significantly higher values than PAs and LNs at all three energies (Figure 4A) $(p<0.05)$. No significant difference was found between LNs and PAs. However, monoenergetic analysis for LNs and PAs showed that their curves crossed at $70 \mathrm{keV}$ (120 kV), where PAs had slightly higher values than LNs below $70 \mathrm{keV}$, and slightly lower values above that energy level (Figure 4A).

In the arterial phase (Figure 4B), LNs showed significantly lower HU values than PAs and the Thy; this was the case at all energies $(p<0.05)$. In addition, there were significant differences between PAs and the Thy at energies of $100 \mathrm{keV}$ and higher; PAs exhibited lower values than the Thy $(p<0.05)$.

In the venous phase (Figure 4C), there was a significant difference between all tissues from 40 $\mathrm{keV}$ to $100 \mathrm{keV}$, with the lowest values evident in LNs, and the highest values evident in the Thy $(p<0.05)$; values for PA lay between the two other structures. From $110 \mathrm{keV}$ and higher, there was no significant difference between LNs and PAs, but significant differences between LNs and the Thy, and between PAs and the Thy.

\section{Discussion}

This study demonstrates that 4D-DECT allows us to distinguish between the characteristics of different tissues, and enhances material characterization when compared with single energy 4D-CT. Consequently, 4D-DECT may help to differentiate pathologies from normal tissue, thus resulting in improved levels of diagnostic accuracy. We identified significant differences in HUvalues between tissues and contrast phases, between spectral curves of the tissues, as well as for Rho, Z, and DEI values. Our results relating to non-contrast scans, which were acquired in DE mode, were particularly novel; these data showed that PAs have lower HU and Rho values than the Thy. Previous studies have also found that PAs and LNs have similar HU values [4]. 
Curves created from HU data over the three contrast phases at different energies were similar in shape; however, the absolute values differed, as expected.

The detection of PAs by 4D-CT is usually performed visually by examining areas in which the PAs are usually situated, as well as looking for ectopic adenomas. PAs are normally identified by the fact that they show clear contrast enhancement in the arterial phase, and washout in the venous phase. However, the discrimination of adenomas from thyroid tissue, especially in patients with goiter, or when adenomas are situated in the thyroid area, is sometimes difficult; therefore, it is important to develop new methods with which to distinguish PAs from other tissues. As shown in Figures 2 and 3, we found a significant difference in attenuation values between the Thy and PAs in non-contrast phase; this might be useful in cases where it is difficult to distinguish between these two structures in the arterial phase and/or venous phase, as well as for cases involving intrathyroidal adenomas.

As clinical investigation is mainly based upon visual judgement, the notable differences in $\mathrm{HU}$ values when compared between different tissues is of significant advantage for diagnostics. As shown in the present study, lower energies, such as $90 \mathrm{kV}$, compared to $150 \mathrm{kV}$ or mixed 0.8, lead to higher HU values; more obvious HU differences between the tissues under investigation may help to improve visual differentiation in clinical scenarios.

Some previous studies reported that the virtual non-contrast (VNC) phase is not useful in DE examinations because the iodine content is subtracted from both PAs and the Thy, leaving both tissues in the same $\mathrm{HU}$ range $[5,9,12]$ in contrast to real non-contrast examinations where the Thy has higher HU values due to its natural iodine content. This concurs with our own observations using VNC images (data not shown). However, when real non-contrast 
images are taken in dual-energy mode, the separation between PAs and the Thy is significant, thus providing another parameter with which to classify tissues and permit their differentiation. Thus, in contrast to Forghani and Mukherji [5], we found that dual-energy data from all three phases are valuable in terms of the quantitative information they provide for the differentiation of tissues. Our arterial phase was taken after 30 seconds; previous studies have used approximately 25 seconds [5,10,12]; furthermore, our venous phase was taken after 75 seconds (compared to 45 - 55 seconds in other studies). Roskies et al. [12] also reported the use of an additional 85 second delay. This might explain why we observed significant differences between all three structures in the venous phase in images taken at 90 $\mathrm{kV}, 150 \mathrm{kV}$, and in the mixed images.

Electron density values, which can be calculated from dual-energy scans, are mainly used to plan radiation therapy. In our study, we found no significant differences between PAs and LNs in the non-contrast phase; this finding concurs with previous research studies which found that the electron density of most normal healthy tissues should be expected to fall in the range of $3.4 \times 10^{23}$ electrons $/ \mathrm{cm}^{3} \pm 5 \%$ [13]. The calculation used by syngo.Via to calculate electron densities is the same as that used in a previous study by Shrimptont [13]. It is important to note that Rho and $\mathrm{Z}$ values are only valid for natural body materials, and that results from materials with higher atomic numbers (such as iodine in the thyroid), or those from a contrast agent, might not be correct, as stated by Siemens in the instructions provided with syngo.Via.

We found that DEI information did not provide any additional value to our tissue analysis because the levels of significance were the same as for the mixed images in the arterial and venous phase; no significant differences were detectable in the non-contrast images. 
Analysis of the spectral curves generated in this study showed that the curves for PAs and LNs crossed each other at an energy of approximately $70 \mathrm{keV}$, indicating that non-contrast examinations for PA diagnostics should not be carried out at energies of $120 \mathrm{kV}$ (approximately $70 \mathrm{keV}$ ); rather, these examinations should be carried out at lower energies, where there is a more evident separation of the curves for PAs and LNs. Information gleaned from such procedures might prove to be crucial in unequivocal cases.

The mean effective dose given to the patients using our protocol was $7.99 \mathrm{mSv}$ (range: 6.4$10.7 \mathrm{mSv})$; this is comparable to the doses used in previous studies $[5,14]$. Our protocol included three phases; this approach is comparable to a protocol previously used for phantom studies and reported by Hoang [15]. In this previous study, the mean dose was $28.5 \mathrm{mSv}$, which was much higher than in other studies, and that of our current study $[5,14]$. The reason for this is because single energy scans were carried out at $120 \mathrm{kV}$ for all three phases. This protocol is suboptimal, not just because of issues related to radiation dose, but also because the differentiation between PAs and LNs, in terms of HU differences, is poorest at $120 \mathrm{kV}$ in noncontrast scans, as demonstrated in our present study (Figure 4A).

As shown in a number of other studies $[3,6,22,11,12,16-21]$, the sensitivity of $4 D-C T$ for PAs is higher (75-92\%) than both ultrasound (40-58\%) and sestamibi scintigraphy (64-75\%). With the additional information of dual-energy data, dual-energy 4D-CT might be considered as the first line preoperative localization imaging test for patients with primary hyperparathyroidism, as recently suggested by Hiebert et al. [6]. 
There are some limitations to our study which should be taken into consideration. First, there is clear potential for selection bias, as we only included cases where standard pre-surgical imaging techniques (ultrasound and sestamibi scintigraphy) were unable to clearly identify PAs, although there was a positive clinical suspicion of parathyroidadenoma. Second, we only had a small number of cases, and our study was retrospective. Finally, ROI placement in small structures, such as small adenomas and lymph nodes, can be challenging. Consequently, the exact location of the ROIs in different contrast phases of the same patient might not always be $100 \%$ accurate.

\section{Conclusion}

Compared with single energy 4D-CT, our data indicate that 4D-DECT is able to reliably identify important spectral tissue characteristics and accessory information. This information may provide additional localization capability for PAs and enhance tissue characterization. By enhancing our ability to differentiate between PAs, LNs, and the Thy, this technique might lead to better diagnostic accuracy. Dual-energy data from non-contrast scans may add value in distinguishing between these three structures and may be particularly useful in equivocal cases. Further validation of our results, using prospective studies, with larger sample sizes, is required.

Conflict of interest: The authors have no conflict of interest in this work

\section{Declaration of interest}

The authors have no conflicts of interest to declare. 


\section{Funding}

This research did not receive any specific grant from any funding agency in the public, commercial or not-for-profit sector.

\section{Acknowledgements}

We thank Petter Quick and Lilian Henriksson for their valuable help in discussing the protocol and performing the examinations. 


\section{References}

[1] I.-L. Nilsson, https://www.internetmedicin.se/page.aspx?id=2738, (n.d.).

[2] G.E.-H. Fuleihan, https://www.uptodate.com/contents/primary-hyperparathyroidismdiagnosis-differential-diagnosis-andevaluation?search=primary\%20hyperparathyroidism\&source=search_result\&selected Title=1 115\&usage_type=default\&display_rank=1, (n.d.).

[3] M.D. Beland, W.W. Mayo-Smith, D.J. Grand, J.T. Machan, J.M. Monchik, Dynamic MDCT for Localization of Occult Parathyroid Adenomas in 26 Patients With Primary Hyperparathyroidism, Am. J. Roentgenol. 196 (2011) 61-65. https://doi.org/10.2214/AJR.10.4459.

[4] K.M. Day, M. Elsayed, M.D. Beland, J.M. Monchik, The utility of 4-dimensional computed tomography for preoperative localization of primary hyperparathyroidism in patients not localized by sestamibi or ultrasonography, Surg. (United States). (2015). https://doi.org/10.1016/j.surg.2014.11.010.

[5] R. Forghani, S.K. Mukherji, Advanced dual-energy CT applications for the evaluation of the soft tissues of the neck, Clin. Radiol. (2018). https://doi.org/10.1016/j.crad.2017.04.002.

[6] J. Hiebert, C. Hague, S. Hou, S.M. Wiseman, The American Journal of Surgery Dual energy computed tomography should be a fi rst line preoperative localization imaging test for primary hyperparathyroidism patients, Am. J. Surg. 215 (2018) 788-792. https://doi.org/10.1016/j.amjsurg.2017.11.048. 
[7] R. Forghani, M. Roskies, X. Liu, X. Tan, A. Mlynarek, R.J. Payne, J.R. Nair, M.P. Hier, M. Levental, Dual-Energy CT Characteristics of Parathyroid Adenomas on 25-and 55Second 4D-CT Acquisitions: Preliminary Experience., J. Comput. Assist. Tomogr. 40 (2016) 806-814. https://doi.org/10.1097/RCT.0000000000000442.

[8] E.D. Roele, V.C.M.L. Timmer, L.A.A. Vaassen, A.M.J.L. van Kroonenburgh, A.A. Postma, Dual-Energy CT in Head and Neck Imaging, Curr. Radiol. Rep. 5 (2017) 19. https://doi.org/10.1007/s40134-017-0213-0.

[9] C. Leiva-Salinas, L. Flors, C.R. Durst, Q. Hou, J.T. Patrie, M. Wintermark, S. Mukherjee, Detection of parathyroid adenomas using a monophasic dual-energy computed tomography acquisition: diagnostic performance and potential radiation dose reduction., Neuroradiology. 58 (2016) 1135-1141. https://doi.org/10.1007/s00234016-1736-4.

[10] N. Seyednejad, C. Healy, P. Tiwari, P. Vos, G. Sexsmith, A. Melck, C. Hague, S.M. Wiseman, Dual-energy computed tomography: a promising novel preoperative localization study for treatment of primary hyperparathyroidism., Am. J. Surg. 211 (2016) 839-845. https://doi.org/10.1016/j.amjsurg.2016.01.003.

[11] D. Lau, H. Yang, P.L. Kei, Dual-energy 4-phase CT scan in primary hyperparathyroidism, Am. J. Neuroradiol. 34 (2013) 2012-2014. https://doi.org/10.3174/ajnr.A3657.

[12] M. Roskies, X. Liu, M.P. Hier, R.J. Payne, A. Mlynarek, V. Forest, M. Levental, R. Forghani, 3-phase dual-energy CT scan as a feasible salvage imaging modality for the identification of non-localizing parathyroid adenomas: a prospective study., J. Otolaryngol. Head Neck Surg. 44 (2015) 44. https://doi.org/10.1186/s40463-015-0098- 
[13] P.C. Shrimptont, Electron density values of various human tissues:, 26 (1981) 907-911.

[14] B. Griffith, H. Chaudhary, G. Mahmood, A.M. Carlin, E. Peterson, M. Singer, S.C. Patel, Accuracy of 2-phase parathyroid CT for the preoperative localization of parathyroid adenomas in primary hyperparathyroidism, Am. J. Neuroradiol. 36 (2015) 2373-2379. https://doi.org/10.3174/ajnr.A4473.

[15] J.K. Hoang, R.E. Reiman, G.B. Nguyen, N. Januzis, B.B. Chin, C. Lowry, T.T. Yoshizumi, Lifetime attributable risk of cancer from radiation exposure during parathyroid imaging: comparison of 4D CT and parathyroid scintigraphy, Am. J. Roentgenol. 204 (2015) W579-W585. https://doi.org/10.2214/AJR.14.13278.

[16] S.J. Brown, J.C. Lee, J. Christie, R. Maher, S.B. Sidhu, M.S. Sywak, L.W. Delbridge, Fourdimensional computed tomography for parathyroid localization: A new imaging modality, ANZ J. Surg. 85 (2015) 483-487. https://doi.org/10.1111/ans.12571.

[17] K.M. Day, M. Elsayed, M.D. Beland, J.M. Monchik, The utility of 4-dimensional computed tomography for preoperative localization of primary hyperparathyroidism in patients not localized by sestamibi or ultrasonography, Surg. (United States). 157 (2015) 534-539. https://doi.org/10.1016/j.surg.2014.11.010.

[18] J.K. Hoang, W. Sung, M. Bahl, C.D. Phillips, How to Perform Parathyroid 4D CT: Tips and Traps for Technique and Interpretation, Radiology. 270 (2014) 15-24. https://doi.org/http://dx.doi.org/10.1148/radiol.13122661.

[19] H.R. Kelly, L.M. Hamberg, G.J. Hunter, 4D-CT for preoperative localization of abnormal parathyroid glands in patients with hyperparathyroidism: Accuracy and ability to 
stratify patients by unilateral versus bilateral disease in surgery-naïve and reexploration patients, Am. J. Neuroradiol. (2014). https://doi.org/10.3174/ajnr.A3615.

[20] A. Mahajan, L.F. Starker, M. Ghita, R. Udelsman, J.A. Brink, T. Carling, Parathyroid fourdimensional computed tomography: Evaluation of radiation dose exposure during preoperative localization of parathyroid tumors in primary hyperparathyroidism, World J. Surg. 36 (2012) 1335-1339. https://doi.org/10.1007/s00268-011-1365-3.

[21] G.J. Randall, P.B. Zald, J.I. Cohen, B.E. Hamilton, Contrast-enhanced MDCT characteristics of parathyroid adenomas, Am. J. Roentgenol. 193 (2009) 139-143. https://doi.org/10.2214/AJR.08.2098.

[22] S.E. Rodgers, G.J. Hunter, L.M. Hamberg, D. Schellingerhout, D.B. Doherty, G.D. Ayers, S.E. Shapiro, B.S. Edeiken, M.T. Truong, D.B. Evans, J.E. Lee, N.D. Perrier, Improved preoperative planning for directed parathyroidectomy with 4-dimensional computed tomography, $\quad$ Surgery. $\quad 140 \quad$ (2006) 932-941. https://doi.org/10.1016/j.surg.2006.07.028. 


\section{Figure legends}

Figure 1: CT images and spectral measurements of a patient with a parathyroid adenoma located in a dorsal position in the right lower part of the right thyroid and examined in three contrast phases; non-contrast (column A), arterial phase (column B), and venous phase (column C). The upper row shows the clinical CT images used for visual assessment (reconstructed with a weighting factor of 0.8 , representing $120 \mathrm{kV}$ images [approximately 70 keV]). Parathyroid adenomas (PAs), thyroid tissue (THY), and lymph nodes (LNs) are highlighted. The second row shows the same patient visualized with color images, generated in the Rho/Z module in syngo.Via. The third row shows spectral measurements (PAs = white, $\mathrm{THY}=$ yellow, $\mathrm{LNs}=$ orange), in which the vertical line in each image corresponds to $70 \mathrm{keV}$ (similar to scans performed at $120 \mathrm{kV}$ ). A particularly point to note is the cross-over of the PA and $\mathrm{LN}$ curves at $70 \mathrm{keV}$ in the non-contrast scans, showing that these two structures cannot be distinguished at this particular energy point. However, the degree of separation increases as the keV decreases. In the arterial phase, the separation between PAs and the THY increases with lower keV, making the visual assessment easier, as can be seen in the fourth row, which shows images at $40 \mathrm{keV}$ in the three phases. 


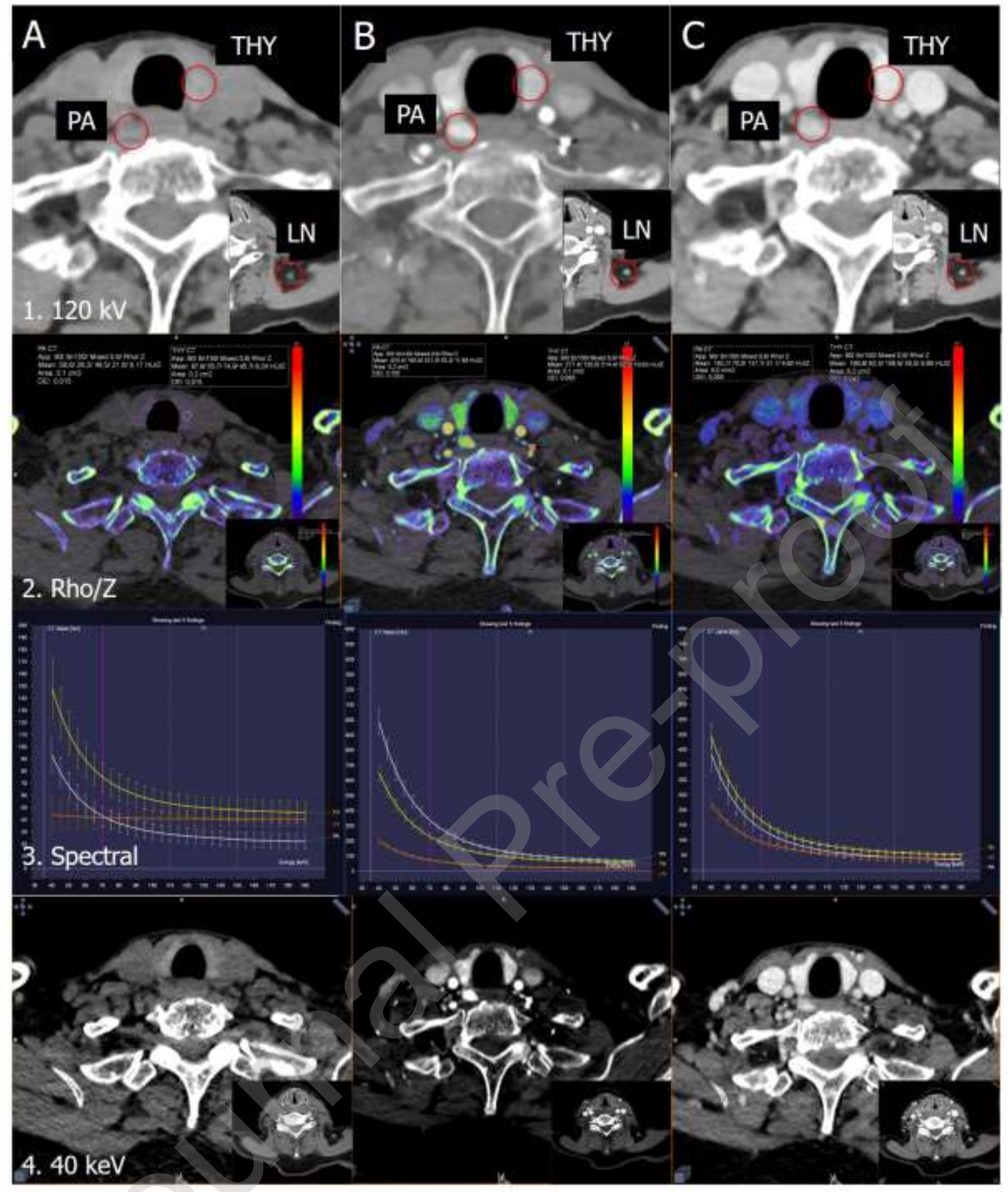

Figure 2: Hounsfield unit (HU) measurements in three tissues (thyroid gland [Thy], lymph nodes [LNs], and parathyroid adenomas [PAs]) in three different contrast phases (withoutcontrast [w/o], arterial [art], venous [ven]), and at three different energies: (A) $90 \mathrm{kV}$, (B) 150 $k V$, and $(C)$ mixed with a factor of 0.8. *significant difference between $L N s$ and PAs $(p=0.05)$; 
**significant difference between PAs and the Thy $(\mathrm{p}=0.05) ; * *$ significant difference between

LNs and the Thy $(p=0.05)$

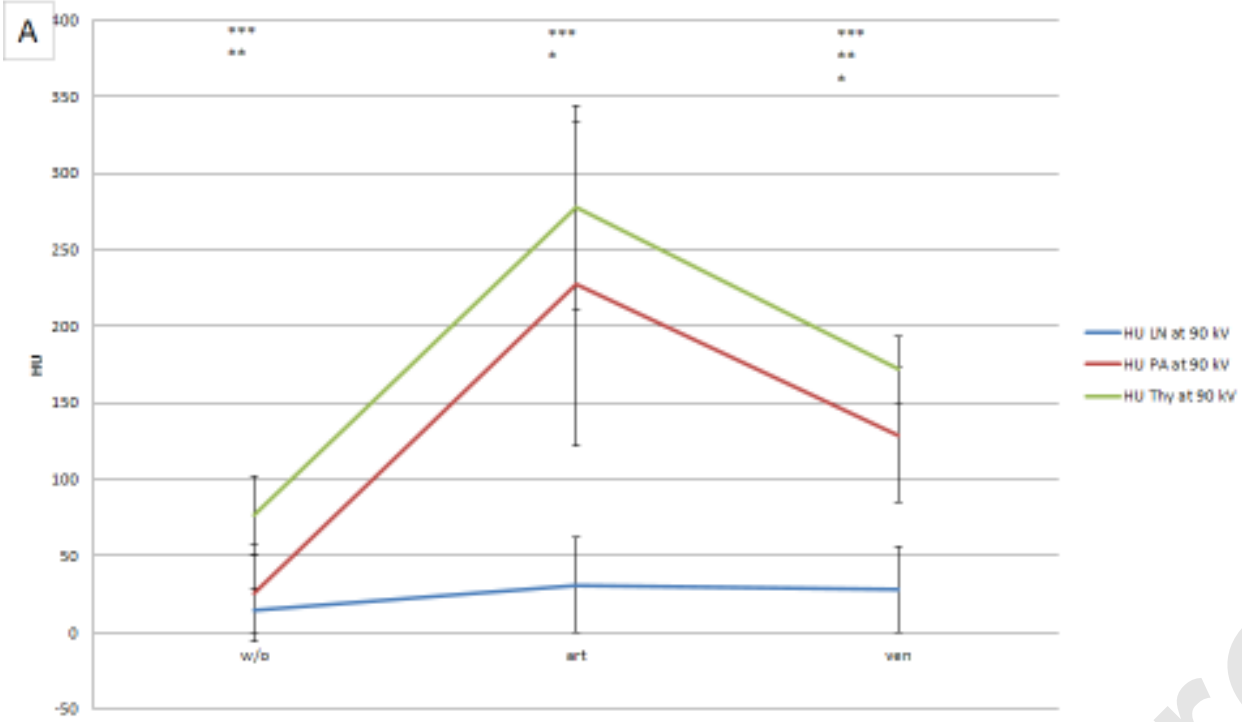

Figure 2 A

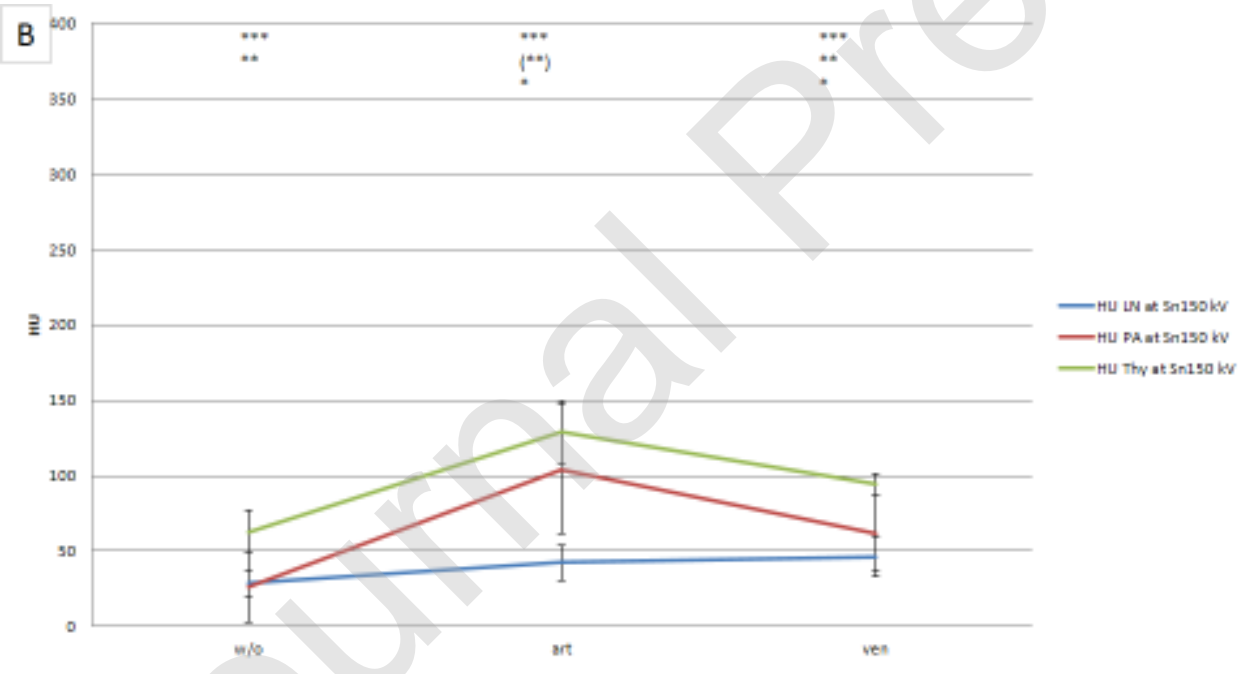

Figure 2 B 


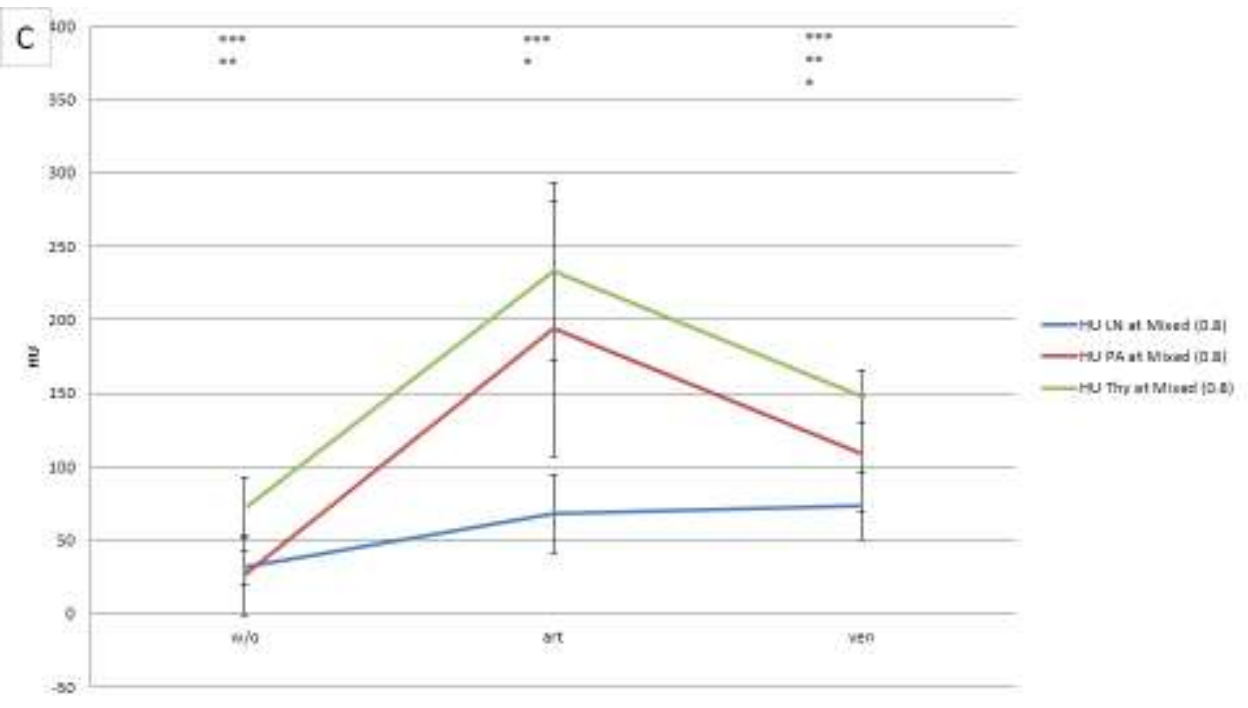

Figure 2C

Figure 3: (A) Measurements of electron density/Rho, (B) atomic number/Z, and (C) dual energy index/DEI in three tissues (thyroid gland [Thy], lymph nodes [LNs], and parathyroid adenomas [PAs]), and in three different contrast phases (without-contrast [w/o], arterial phase [art], and venous phase [ven]). *significant difference between $L N s$ and PAs $(p=0.05) ;{ }^{* *}$ significant difference between PAs and the Thy $(p=0.05) ; * * *$ significant difference between LNs and the Thy $(p=0.05)$ 


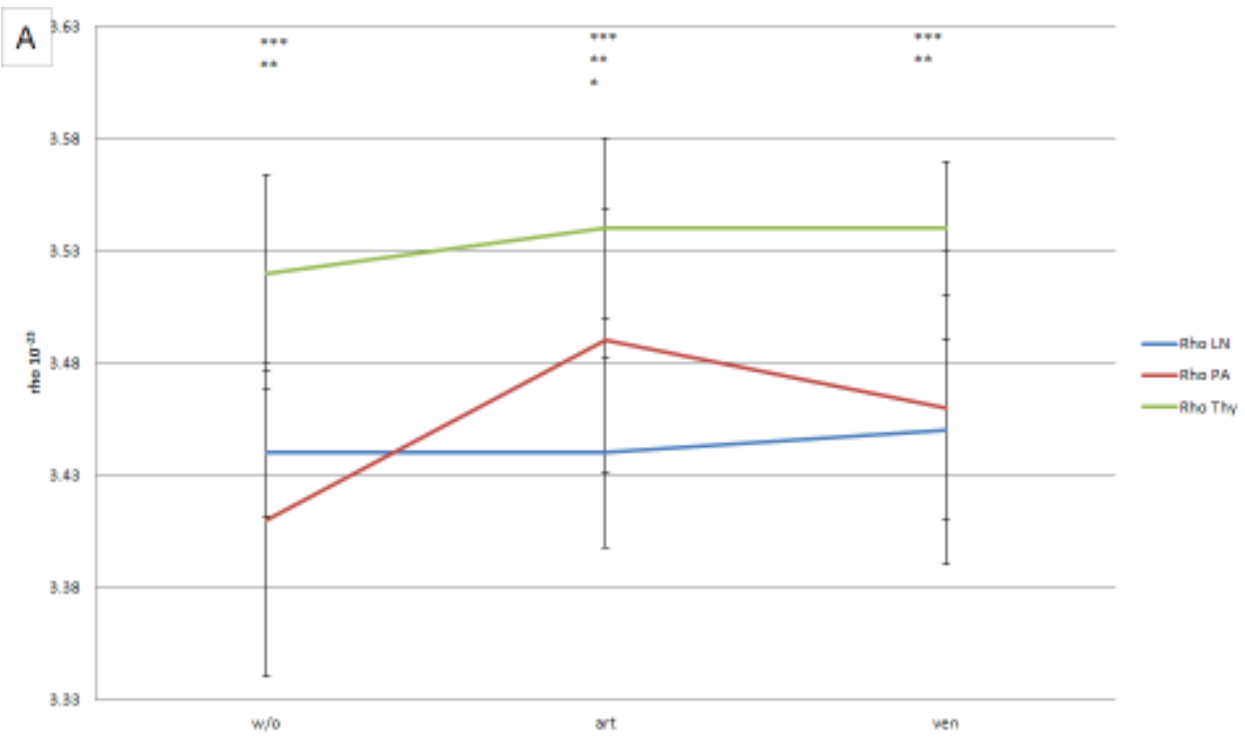

Figure $3 \mathrm{~A}$

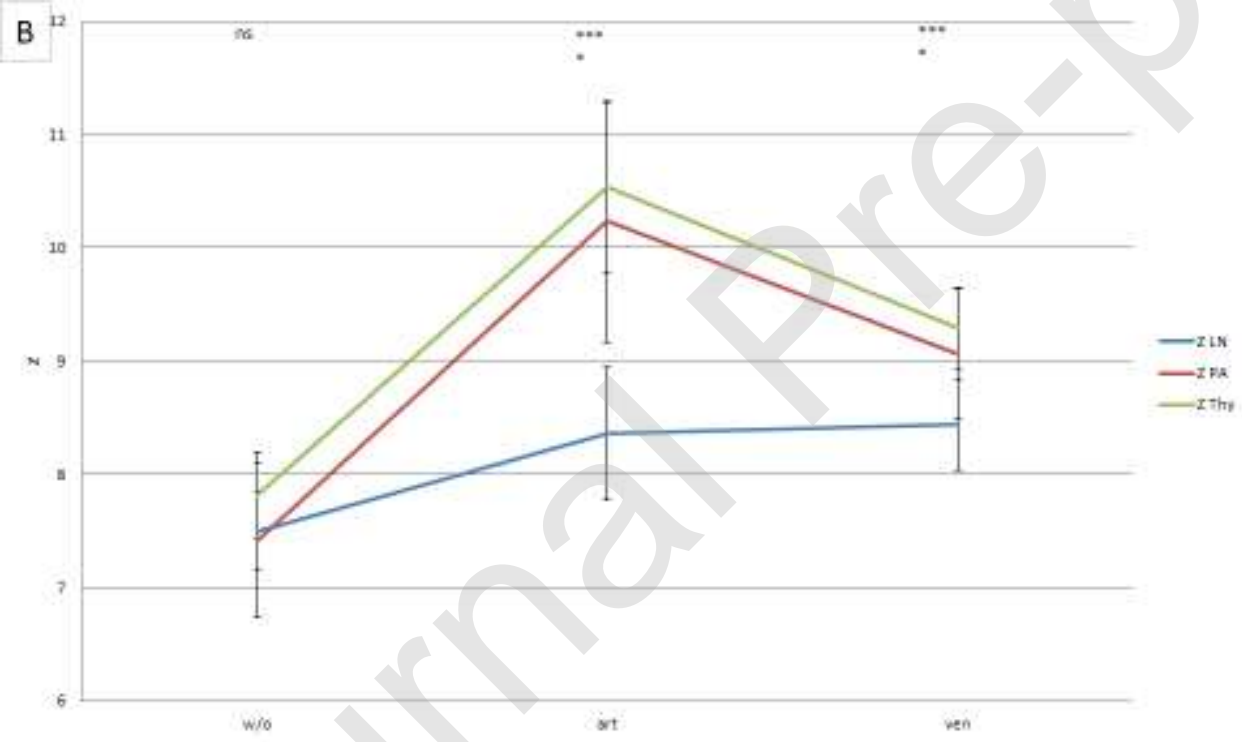

Figure 3 B 


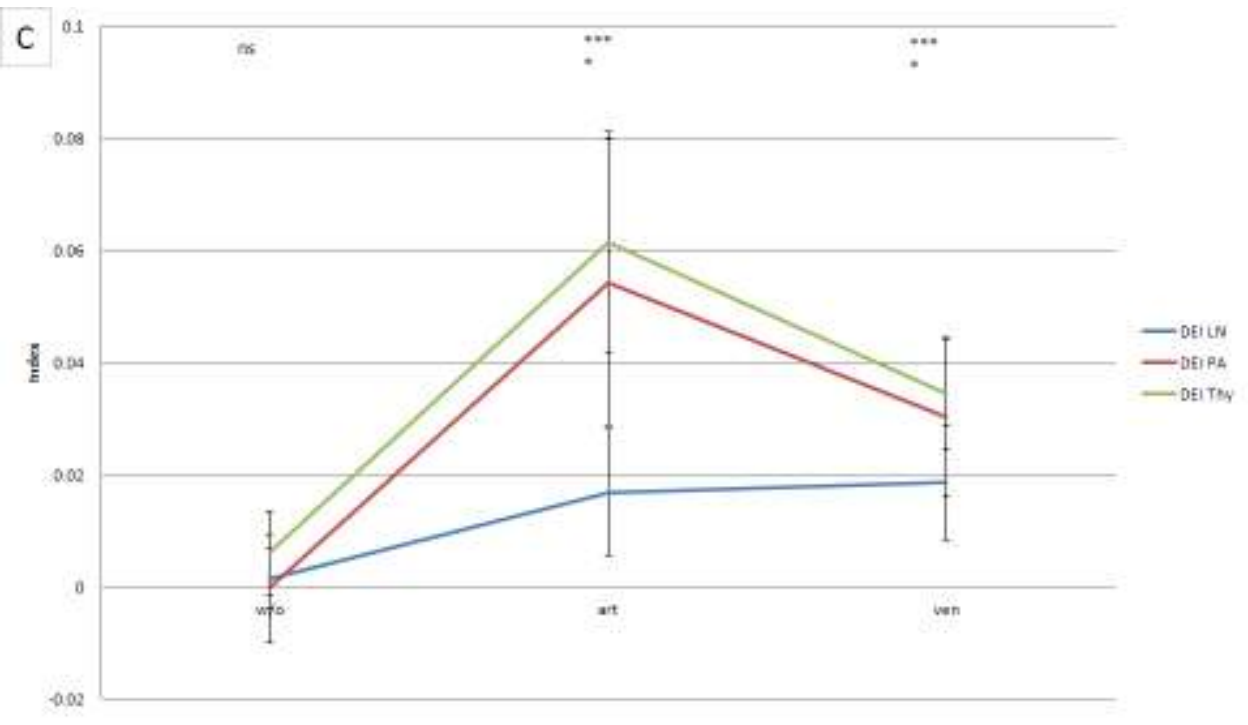

Figure $3 \mathrm{C}$

Figure 4: Monoenergetic curves of three tissues (thyroid gland [Thy], lymph nodes [LN], and parathyroid adenomas [PA]) in three different contrast phases: (A) without-contrast [w/o], (B) arterial phase [art], and (C) venous phase [ven]). *significant difference between LNs and PAs $(p=0.05) ;{ }^{* *}$ significant difference between PAs and the Thy $(p=0.05) ;{ }^{* * *}$ significant difference between LNs and the Thy $(p=0.05)$ 


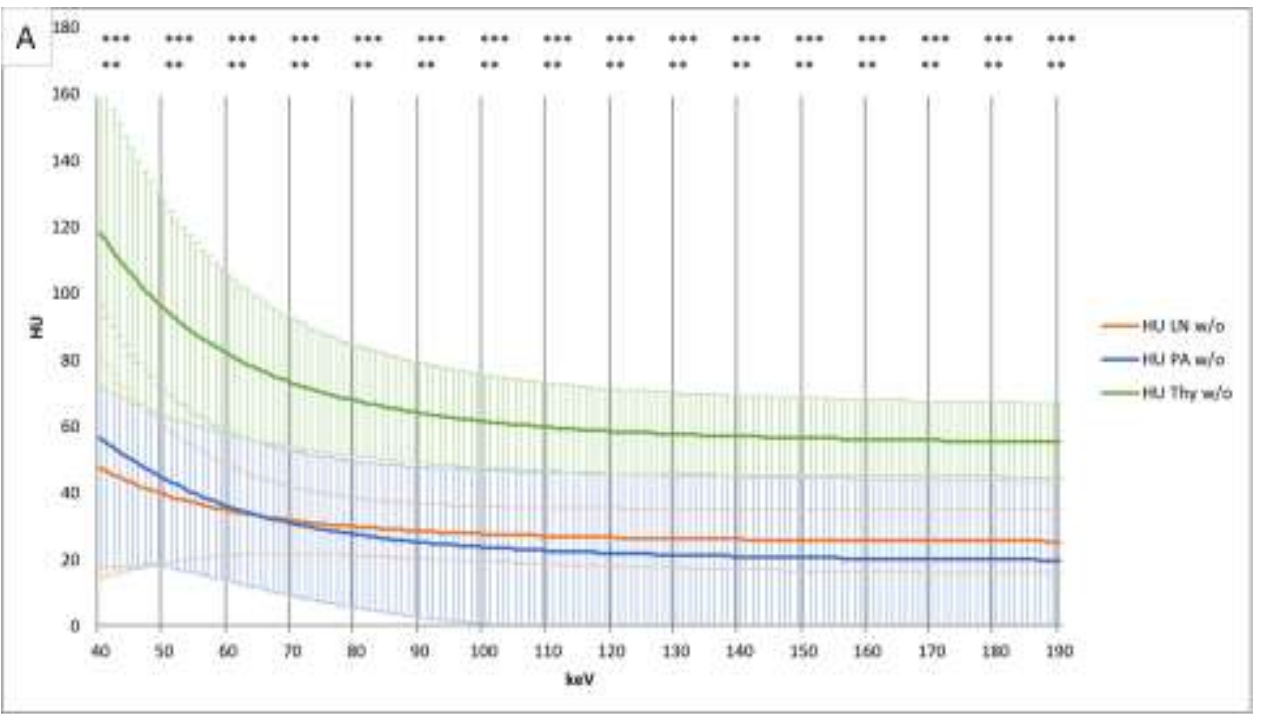

Figure $4 \mathrm{~A}$

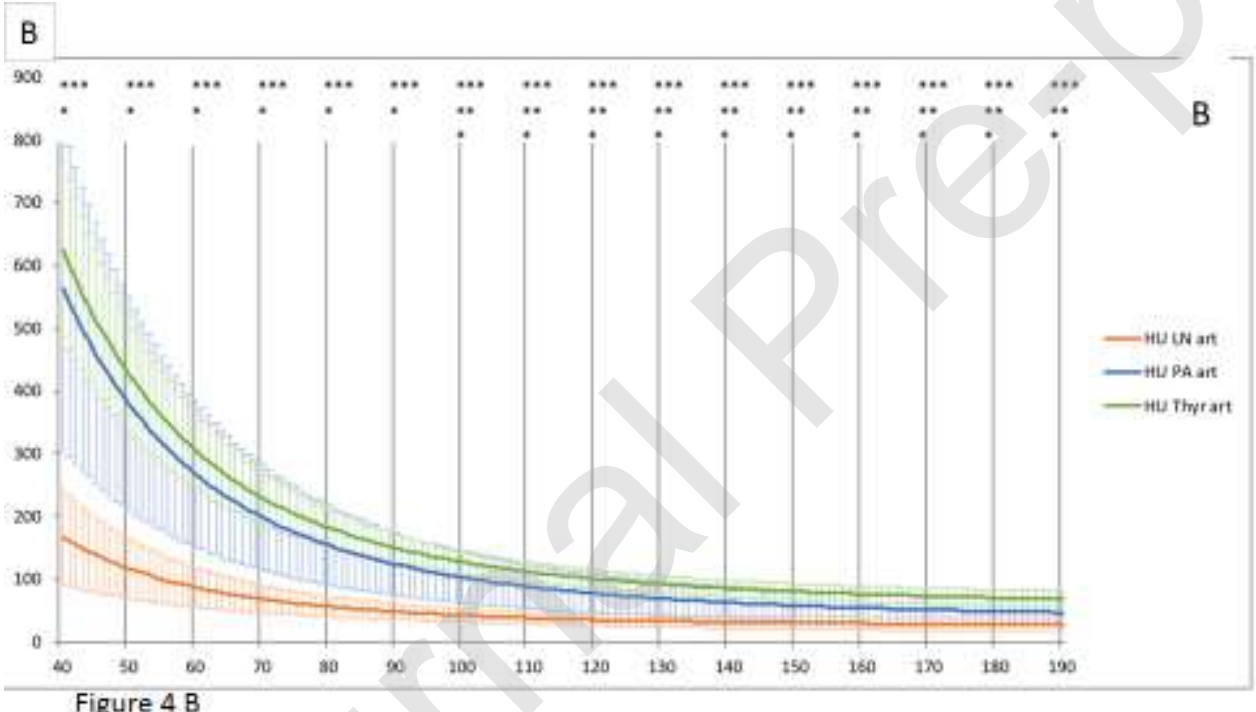




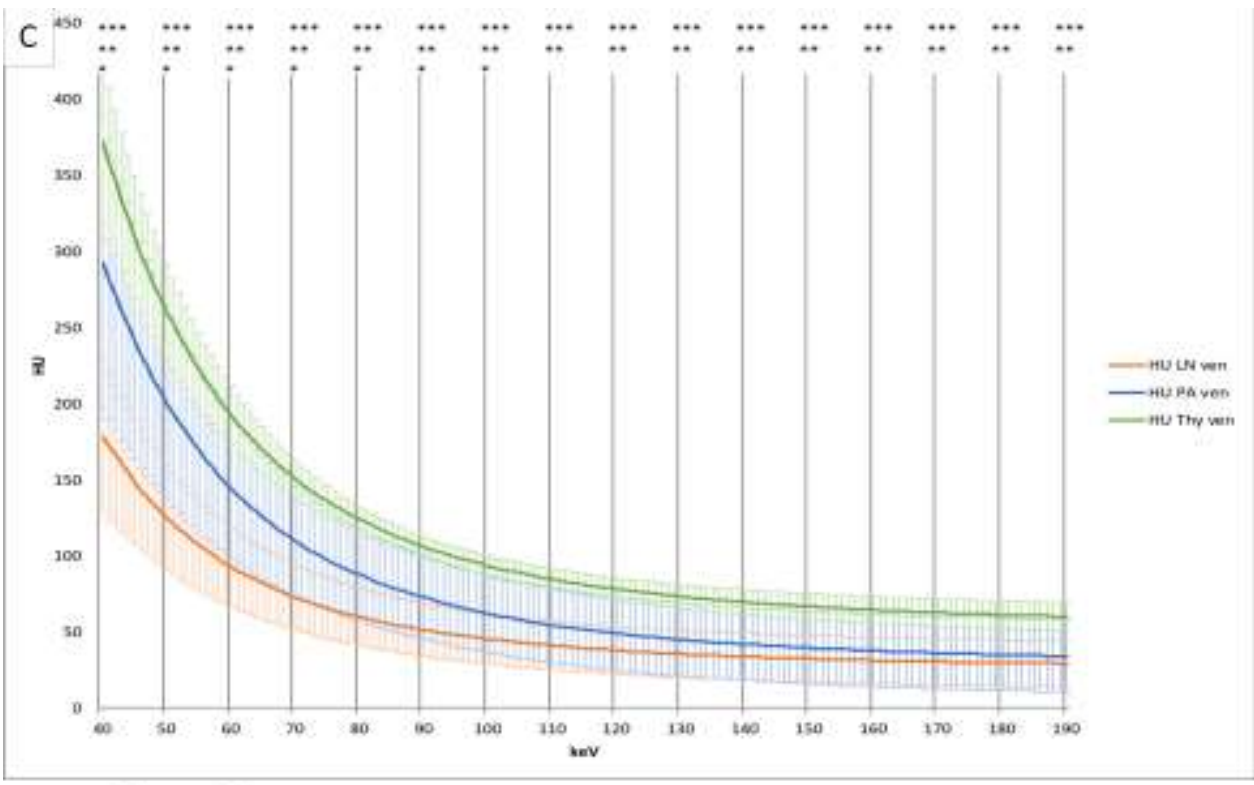

Figure $4 \mathrm{C}$ 